\section{ACTION OF LOCAL HORMONES}

$\mathrm{O}^{2}$

February 16 there was a discussion at the Royal Society on the action of local hormones. In opening, Prof. J. H. Burn, professor of pharmacology in the University of Oxford, said that the first proposal was to discuss the mechanism of the action of drugs. This subject was too wide, and it was felt better to discuss instead the more recent knowledge of the action of adrenaline, acetylcholine and histamine.

Both Feldberg in 1930 and Gaddum in 1936 had referred to adrenaline, acetylcholine and histamine as local hormones, but the concept that these substances control local activity had been forgotten owing to the importance they had acquired as transmitters of nerve impulses. Prof. Burn then gave an illustration of the function of acetylcholine as a local hormone in the auricles of the rabbit heart. He said that evidence has been obtained that acetylcholine not only inhibits the auricles, but also in some circumstances stimulates them. Thus, when auricles are exposed to 'Paludrine', after a time they cease to beat, but can be started again by acetylcholine. Similarly, if the auricles are allowed to beat for $24 \mathrm{hr}$. or more until they stop, the beat can be started by acetylcholine. These observations led to a study of the synthesis of acetylcholine by an acetone powder prepared from auricles. Feldberg and Mann worked out a method for studying the synthesis in brain, and Comline, using this method, found synthesis to occur in auricles. The acetone powder was incubated with choline and citrate in the presence of adenosine triphosphate and other substances. A comparison was made by Dr. Edith Bülbring between powder from freshly excised auricles, powder from auricles which had stopped beating in $24 \mathrm{hr}$., and powder from auricles in which the beat was started again by acetylcholine. It was found that the stopped auricles had lost most of the synthetic power of the fresh auricles, but that the re-started auricles had regained it. Further, it was found that just as acetylcholine depresses the contractions of fresh auricles in a bath, so the addition of acetylcholine to tubes containing powder from fresh auricles depresses the synthesis in proportion to the amount added. More surprising was the observation that the addition of acetylcholine to tubes containing powder from stopped auricles increases the synthesis in proportion to the amount added, exerting an autocatalytic effect. This again is parallel to the action of acetylcholine in stimulating the contraction of stopped auricles in the bath. Thus there is at all stages an extremely close parallelism between the size of the contractions and the power to synthesize acetylcholine. To explain the function of acetylcholine in relation to the contractions, Prof. Burn suggested that its role is similar to its function in skeletal muscle, where the motor nerve liberates acetylcholine to cause the contraction. In cardiac muscle, acetylcholine seems to be continuously synthesized and then liberated to maintain the rhythmicity. This is its function as a local hormone.

The action of acetylcholine in the blood vessels was then discussed, since it has certain points of similarity to the action in the auricles.

Dr. W. Feldberg, of the National Institute for Medical Research, spoke on the origin and function of the acetylcholine in the intestinal wall, where acetylcholine is continually produced and liberated. The nerve cells have been thought to produce it, but various pieces of evidence now indicate that this is not so. In the perfused superior cervical ganglion, the liberation of acetylcholine by preganglionic stimulation is prevented by cocaine; but in the intestine, cocaine, even in high concentration, does not prevent the liberation.

The synthesis of acetylcholine by different parts of the intestinal wall, glandularis mucosa, muscularis mucosa, submucous coat and the external muscular coats has been carefully investigated, and it has been found that the distribution of the synthesizing enzyme bears no relation to the localization of the nerve cells. These facts suggest that the acetylcholine production is not nervous in origin.

If acetylcholine is produced in non-nervous tissue, what is its function? Dr. Feldberg suggested that, in the first place, by its action on smooth muscle fibres, the released acetylcholine will increase muscle tone and motility and so facilitate the peristaltic reflex. In the second place, by its action on the. gland cells, it will stimulate the secretion of succus entericus; it may also influence absorption. In the third place, acetylcholine may stimulate the nerve cells so as to keep them in a continuous state of tonic activity, or in such a way as to lower their threshold to other stimuli. Ambache has published experiments which on analysis indicate that 5-20 per cent of the effect of acetylcholine on the small intestine results from ganglionic stimulation. Dr. Feldberg thought that if such a mechanism exists in the wall of the intestine, it may also exist in the central nervous system.

A later contribution to the discussion by Prof. R. H. S. Thompson, professor of chemical pathology in Guy's Hospital Medical School, London, may be suitably considered here. An investigation of the distribution of the two main types of mammalian cholinesterase, called by Mendel "true" and "pseudo", has revealed a suggestive contrast in their distribution in different tissues. Both enzymes destroy acetylcholine; true cholinesterase will also split ecetyl. $\beta$-methylcholine but not benzoylcholine, whereas pseudocholinesterase will split benzoylcholine but not acetyl- $\beta$-methylcholine. Prof. Thompson said that in brain, in skeletal muscle and in the suprarenal gland, the enzyme present has been found to be predominantly true cholinesterase; in stomach, liver, lung and salivary gland the amounts of the two enzymes are similar; finally, in the auricle and ventricle of the heart, in the intestinal muscle and mucosa, and in Harderian gland and skin, the pseudocholinesterase is present in much greater amount than the "true". In the human placenta, after perfusion to remove red cells and plasma, the true cholinesterase predominates. Since the plecenta is free from nervous tissue, it is clear that true cholinesterase is not specifically associated with nervous activity.

Prof. J. H. Gaddum, professor of pharmacology in the University of Edinburgh, discussed the identity of the substances liberated by sympathetic stimulation and their actions. He pointed out that the primary amine noradrenaline is present, with adrenaline, in extracts of adrenal glands or adrenal medullary tumours. Both substances are liberated from the adrenals by stimulation of the splanchnic nerves. Extracts of adrenergic nerves also contain both substances, and a mixture is liberated when these nerves are stimulated. In extracts of splenic nerves and in blood collected from the splenic vein during stimulation of these nerves, there is at least ten times more noradrenaline than adrenaline. The 
factors controlling the percentage of mothylation are not yet fully elucidated.

Most of the actions of these two drugs aro quali. tatively the samo but quantitatively different. Barger and Dale divided effects into excitor effects and inhibitor offects. In the light of recent evidence (Konzett, West, Ahlquist) this classification must now be modified. Various excitor effects and the inhibitor effect on the intestine may be classed together as $\alpha$-offects, and various inhibitor effects and the quickening of the heart may be classed as $\beta$-effects. Like other antagonists, ergotoxino in small doses may cause sensitization. Local hormones may also have opposed effects in different doses. Inhibitory effects after large doses may be comparable with a similar effect of some substrate on enzymes, or with adaptation of sense organs, or with accommodation in excitable tissues, or it may be associated with more or less continuous depolarization of surfaces.

Prof. R. A. Peters, Whitley professor of biochemistry in the University of Oxford, described work which has beon done on fluoroacetate poisoning. Following on observations in vitro with kidney homogenates, determinations of citrate have been made in various organs of the rat after injections of fluoroacetate which induced convulsions. Within an hour, very high accumulations of citrate were found, which can bo: explained in terms of the tricarboxylic acid cycle. It is clearly possible, he said, that fluoroacetate can be activated like acetate and built in to form a fluoro-6-carbon acid which may be too foreign for furthor metabolism. There is reason to think that the block is at the stage of isocitric acid oxidation, and that it is induced by the inhibitory action of a fluoro6-carbon acid. The accumulation of citric acid has been ostablished by its isolation. Prof. Peters suggested that increases and decreases of citrate formed in tissue cells may be one of the regulators of ionic calcium activity. Bronk and his colloagues have shown the effects of sodium citrate in increasing the excitability of nerve, and thus the occurrence of convulsions after injection of fluoroacetate may be explained. It has been possible to show, by examining the brachial and sacral plexus twelve hours after injecting fluoroacetato, that citrate can accumulate in frog's nerve. Normal activity may therefore be controlled in part by the interaction of ionic calcium with citrate produced by the tricarboxylic acid cycle.

Prof. G. L. Brown, Jodrell professor of physiology at University College, University of I London, reviewed the action of acetylcholine and adrenalino in striped muscle in order to consider whether their excitatory and inhibitory effects in cardiac and smooth muscle can be similarly explained. When acetylcholine is applied to the end plate of skeletal muscle, it generates a potential which starts the propagated excitation wave. If acetylcholine persists in the presence of an anticholinesterase, there is prolonged depolarization and repetitive firing of the muscle fibre. Persistonce of high concentrations at the mammalian end plate can produce neuromuscular block due to depolariza. tion which does not evoke propagated contractions, but a localized contracture which blocks conduction along the muscle fibre. In smooth muscle, acetylcholino does appear to act by depolarizing some sensitive rogion and initiating a contractile process.

Is it possible to explain the vagal inhibition of the heart as due to excess of acetylcholine? Gaskell found that when the potential differenco betweon the middle of tho tortoise auricle and the damaged end was measured, vagus stimulation increased the resting potential.
Hence the direction of the effect was the opposite of what would be expected if conduction block were produced by depolarization in a conducting pathway.

Adrenaline has two actions on skeletal muscle, namely, a decurarizing action at the neuromuscular junction and a diroct action on the contractile process whereby an increase in muscle tension is associated with a retardation of the spread of the action potential and an increase in tho rosting potential. In smooth muscle, adrenaline acts very like acetylcholine in skeletal muscle. On the nictitating membrane, adrenaline produces a local depolarization from which arise in turn propagated muscle responses with action-potential spikes. Injected adrenalino, again, can produce a local non-propagated depolarization and a contracture which prevents the propagation of excitation arising from incident nervo volleys. Adrenaline must be regarded as an agent capable of evoking locel potentials at the sensitive regions of the structure concerned.

The action of adrenaline on the heart seems to have no connexion with its action in skeletal muscle. There is no satisfactory evidence that the inhibitory actions of adrenaline are due to the accumulation of a substance which in lower concentration is excitatory. Bozler's recent work suggests that the inhibitory actions of adronaline on the gastro-intestinal tract may eventually be correlated with its actions in skelotal muscle.

Prof. Z. M. Bacq, of Liège, considered that it is important to know what physiological function a substance found in the body exerts before it is called a hormone. Thus pure adrenaline is present in the parotoid glands of toads, but its function is unknown. He pointed out that acetylcholine has other functions besides those of a transmitter of nervo impulses, since in crustaceans it plays no part in the synapse, though it protects the ganglion against strychnine poisoning. Further, he said that adronochrome is equally as important as adrenaline. The long-lasting effect of adrenaline on bleeding time is probably due to the adrenochrome into which it is converted. Recent observations with the electron microscope suggest, he said, that the neurotubules in nerve may transmit a constant though vory slow flow of local hormones into the innervated tissue.

Dr. H. K. F. Blaschko, senior research officer in the Department of Pharmacology, University of Oxford, discussed analogies between enzyme-substrate relationship and the affinity of local hormones for the excitablo tissue. Enzymes which show stereochemical specificity for one steric configuration are assumed in the enzymic reaction to have a fixed spatial arrangement, relative to the enzyme, of three at least out of the four groups attached to the asymmetric carbon atom. This was discussed for the bacterial and mammalian L-amino-acid docarboxylases which form adrenaline-like amines (tyramine, hydroxytyramine, noradrenaline) from the corresponding $L$-amino-acids. Similarly, the specific action of lævoadrenaline must be duo to an engagemont of three of the four groups surrounding the asymmetric carbon atom. These are: the catechol group, the basic group and the aliphatic hydroxyl group. The lesser activity of dextro-adrenaline is explained by assuming that only the catechol and basic groups are engaged, but that the hydroxyl group is not. The same mode of attachment occurs in opinine, a compound in which the aliphatic hydroxyl group of adronaline is replaced by hydrogen; this compound is about as active as doxtro-adrenaline. 